\title{
Giant Parathyroid Adenoma: A Case of Primary Hyperparathyroidism
}

\author{
Jasmin N Cabrera ${ }^{1}$, Julia Rodríguez ${ }^{2}$ and Sylvia Batista ${ }^{3 *}$ \\ ${ }^{1}$ General Physician, CEDIMAT, Santo Domingo, Dominican Republic \\ ${ }^{2}$ Knowledge Management and Epidemiology, CEDIMAT, Santo Domingo, Dominican Republic \\ ${ }^{3}$ Endocrine Surgeon, CEDIMAT, Santo Domingo, Dominican Republic
}

\begin{abstract}
Introduction: We report an adult male with the sole condition of recurrent nephrolithiasis, with no other associated symptoms nor family history, who was later diagnosed with Primary Hyperparathyroidism due to a giant right superior parathyroid adenoma. To the best of our knowledge, this is one of the few well-documented cases of this rare presentation in a male patient of the Dominican Republic. This case should affect the practice of physicians who interact with primary hyperparathyroidism and are involved in their management.
\end{abstract}

\begin{abstract}
Case presentation: A 55-year-old hispanic man who was diagnosed and treated for the last 8 years for recurrent calcium nephrolithiasis with no other associated symptoms. Etiology for his condition remained unknown to the patient until he visited a tertiary care level hospital for routine check-up, where he was referred to endocrinology due to incidental biochemical findings of elevated PTH levels $(718.10 \mathrm{pg} / \mathrm{ml})$, hypercalcemia $(13.27 \mathrm{mg} / \mathrm{dl})$ ), elevated ALP (386.90 U/L), 24 $\mathrm{hr}$ urinary calcium of $483.04 \mathrm{mg} / 24 \mathrm{hr}$, and hypophosphatemia $(2.03 \mathrm{mg} / \mathrm{dl})$. Other symptoms common to PHPT such as abdominal pain, fatigue, weakness, psychiatric disturbances such as depression, or numbness of the skin were denied by the patient. Family history was negative for thyroid, parathyroid or kidney disease. The patient works frequently outdoors and is constantly exposed to the sun. The patient underwent a standard Tc99m-sestamibi scan that revealed heterogenous delayed persistent tracer localization in the right upper parathyroid region. An ultrasound of the neck was performed which revealed a right superior mass of the parathyroid gland measuring $4.00 \times 2.48 \times 2.02 \mathrm{~cm}(700 \%$ increase in size) and weighting $10.6 \mathrm{gm}$.
\end{abstract}

\begin{abstract}
Conclusion: Giant parathyroid adenomas are rarely encountered among patients with primary hyperparathyroidism and are known to present with distinct clinical and biochemical features that differ from typical parathyroid adenomas. To the best of our knowledge, this case report describes the first case reported in the Dominican Republic of a patient with Giant Parathyroid Adenoma (GPTA). This case report also reviews the published literature to report on the clinical characteristics and typical presentation of GPTA as well as diagnosis and treatment.
\end{abstract}

\section{Keywords}

Parathyroid adenoma, Primary hyperparathyroidism, Hypercalcemia

\section{Introduction}

We report an adult male with the sole condition of recurrent nephrolithiasis, with no other associated symptoms nor family history, who was later diagnosed with Primary Hyperparathyroidism due to a giant right superior parathyroid adenoma. To the best of our knowledge, this is one of the few well-documented cases of this rare presentation in a male patient of the Dominican Republic. This case should affect the practice of physicians who interact with primary hyperparathyroidism and are involved in their management. Primary hyperparathyroidism is a pathology of the parathyroid glands, four pea-sized glands that are located on or near the thyroid gland in the neck. "Primary" refers to the disorder originating from the parathyroid glands, rather than resulting from another health problem such as kidney failure. A Giant parathyroid adenoma (GPTA) is a rare cause of primary hyperparathyroidism and usually presents symptomatically with high calcium and PTH levels. The pathophysiology of PHPT is autologous secretion of parathyroid hormone (PTH) by

*Corresponding author: Sylvia Batista, Endocrine Surgeon, CEDIMAT, Santo Domingo, National District, Dominican Republic

Accepted: August 25, 2021

Published online: August 27, 2021

Citation: Cabrera JN, Rodríguez J, Batista S (2021) Giant Parathyroid Adenoma: A Case of Primary Hyperparathyroidism. J Surgical Endocrinol 3(2):97-100 
one or more of the parathyroid glands. Although PHPT can be caused by parathyroid hyperplasia or carcinoma, around $85 \%$ of cases of PHPT are due to PTAs, and the majority of these are because of solitary PTAs, of which GPTA comprise a small number. The normal parathyroid gland weighs approximately 50-70 mg. Parathyroid adenomas (PTAs) are usually small, measuring $<2 \mathrm{~cm}$ and weighing $<1 \mathrm{gm}$. According to an article by the UCLA Endocrine Center, a GPTA, although rare, are most commonly defined as weighing $>3.5 \mathrm{gm}$, with some cases reporting weights up to $110 \mathrm{gm}$. Both PTA and GPTA present with primary hyperparathyroidism (PHPT), the third most common endocrine disorder. Giant parathyroid adenoma is typically diagnosed by imaging and laboratory studies. Due to the rarity of GPTAs, their management requires surgical expertise, aiming at complete resection. This case should affect the practice of physicians who interact with primary hyperparathyroidism and are involved in their management [1-4].

We report a case of a 55-year-old man who developed recurrent nephrolithiasis and osteoporosis due to severe primary hyperparathyroidism associated with a Giant Parathyroid Adenoma (GPTA). This case report follows the CARE Guidelines.

\section{Narrative}

A 55-year-old hispanic man who was diagnosed and treated for the last 8 years for recurrent calcium nephrolithiasis with no other associated symptoms. Etiology for his condition remained unknown to the patient until he visited a tertiary care level hospital for routine check-up, where he was referred to endocrinology due to incidental biochemical findings of elevated PTH levels $(718.10 \mathrm{pg} / \mathrm{ml})$, hypercalcemia ( $13.27 \mathrm{mg} / \mathrm{dl})$, elevated ALP (386.90 U/L), $24 \mathrm{hr}$ urinary calcium of $483.04 \mathrm{mg} / 24 \mathrm{hr}$, and hypophosphatemia (2.03 $\mathrm{mg} / \mathrm{dl})$. Other symptoms common to PHPT such as abdominal pain, fatigue, weakness, psychiatric disturbances such as depression, or numbness of the skin were denied by the patient. Family history was negative for thyroid, parathyroid or kidney disease. The patient works frequently outdoors and is constantly exposed to the sun. An ultrasound of the neck was performed which revealed a right superior mass of the parathyroid gland measuring $4.00 \times 2.48 \times 2.02$ $\mathrm{cm}$ (700\% increase in size) and weighting $10.6 \mathrm{gm}$. The patient underwent a standard Tc99m-sestamibi scan that revealed heterogenous delayed persistent tracer localization in the right upper parathyroid region. Bone densitometry (DEXA) reported a T-score of -2.6 , consistent with osteoporosis. The

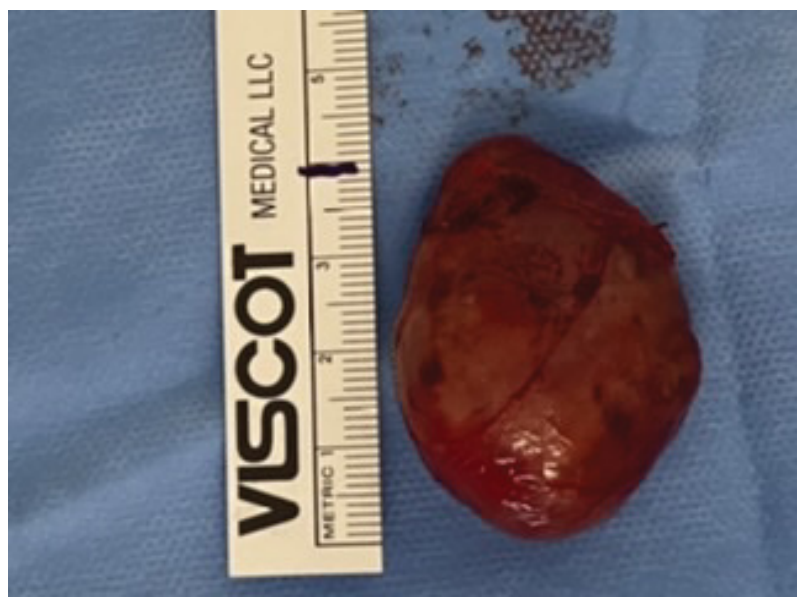

Figure 1: Right superior giant parathyroid adenoma measuring $4.00 \times 2.48 \times 2.02$.

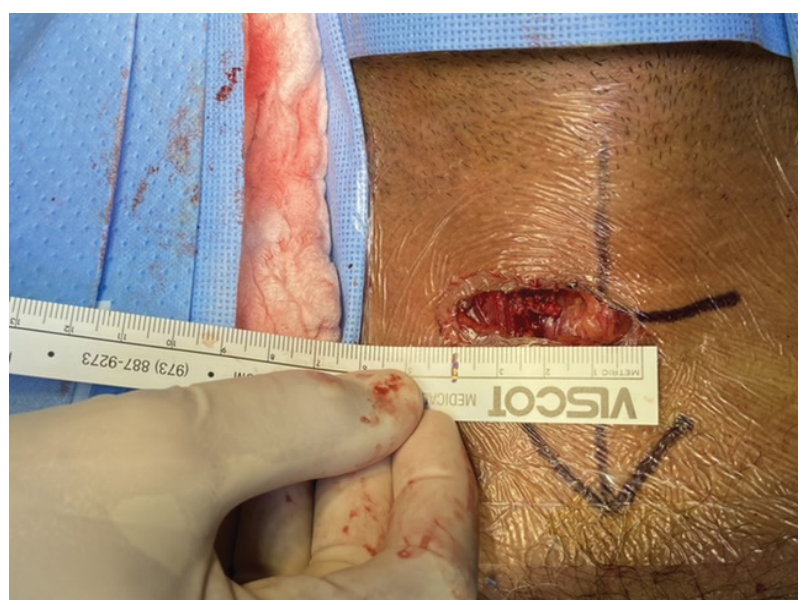

Figure 2: $4 \mathrm{~cm}$ minimal invasive parathyroidectomy incision. 
highly specific findings reported in the ultrasound allowed the surgeon to perform a minimally invasive selective approach with a smaller incision, in which the abnormally enlarged gland was located, dissected and removed safely. Transquirurgic PTH levels were measured, reporting a decrease to $75.47 \mathrm{pg} / \mathrm{ml}$. The enlarged gland was sent for frozen section, reporting scattered hyperchromatic nuclei exhibiting atypia, and no morphologic features of a parathyroid carcinoma with no capsular or vascular invasion. This was confirmed by the final immunohistochemistry studies, which reported positive for PTH and negative for thyroglobulin and calcitonin, as well as a $\mathrm{KI}-67<3 \%$ (consistent with low proliferation). There were no surgical complications and the patient achieved a complete surgical recovery. He was placed subsequently on oral calcium and vitamin D supplementation thrice daily for prevention of hungry bone syndrome and analgesia. During the follow-up consultation 7 days post-surgery, laboratories demonstrate hypocalcemia $(8.85 \mathrm{mg} / \mathrm{dl})$ with elevated PTH $(150.30 \mathrm{pg} / \mathrm{ml})$ as a manifestation of Hungry bone syndrome, however the patient remained asymptomatic. The dose of the calcium supplement was increased and outpatient follow-up continues (Figure 1, Figure 2, Figure 3 and Figure 4). Source: Sylvia Batista

\section{Discussion}

Parathyroid adenomas weighting > $3.5 \mathrm{gm}$ with size $>3 \mathrm{~cm}$ are commonly termed as giant parathyroid adenomas, and this is a rarity in literature. GPTA present less than $10 \%$ of parathyroid adenomas. Usually, both patients with parathyroid carcinoma as well as patients with giant parathyroid adenoma present severe hypercalcemia with pathologies secondary to excessive elevations of PTH, hypercalcemia, and high alkaline phosphatase. According to the literature, there is a correlation between the weight of

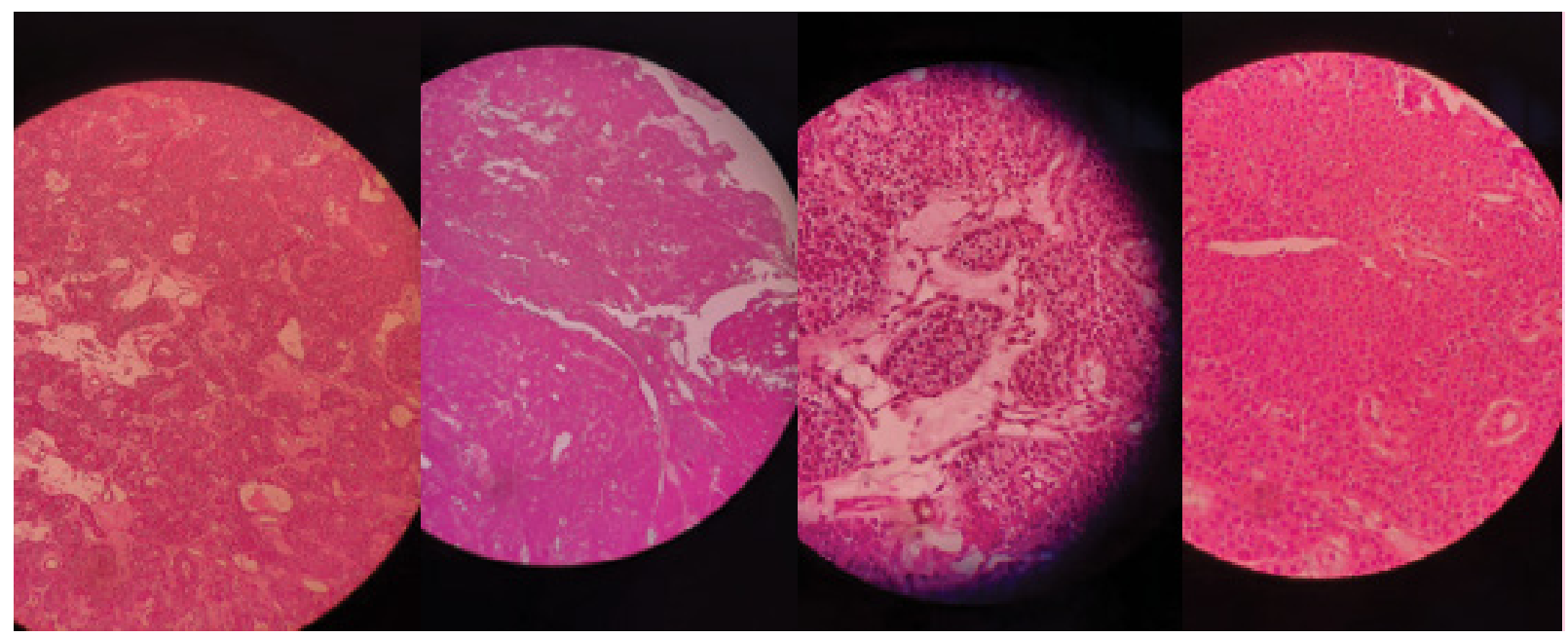

Figure 3: Various cuts of encapsulated parathyroid adenoma, presenting scattered hyperchromatic nuclei exhibiting atypia, and no morphologic features of a parathyroid carcinoma with no capsular or vascular invasion.

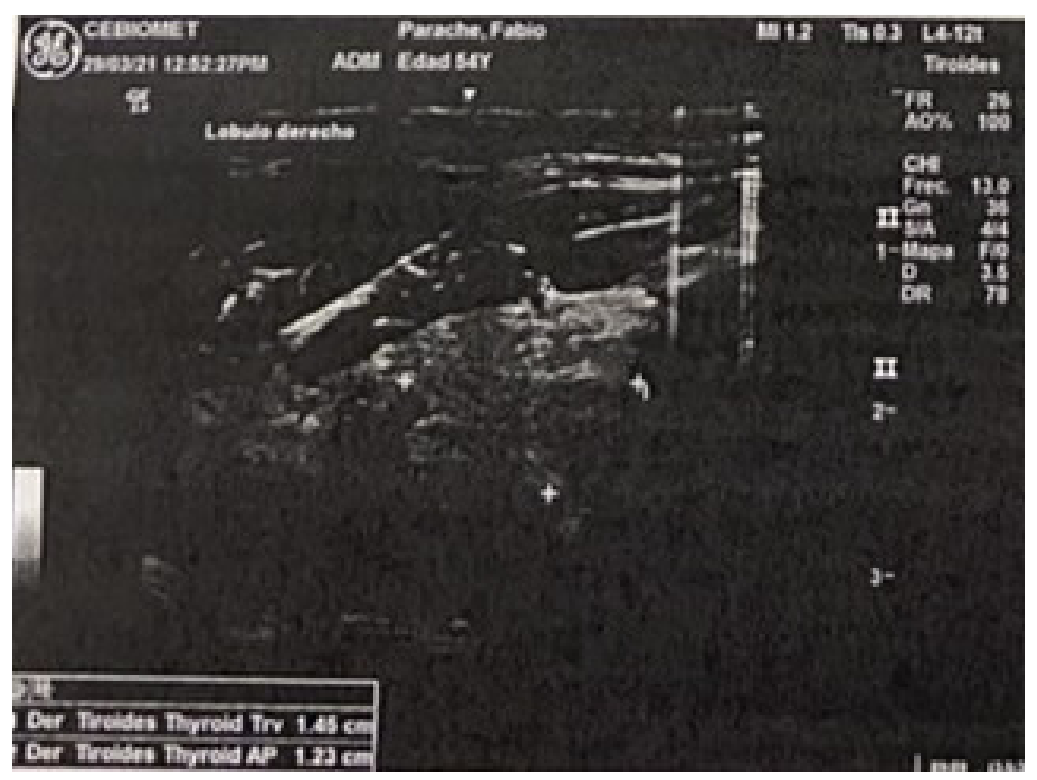

Figure 4: Neck ultrasound revealing $4.00 \times 2.48 \times 2.02$ right superior giant parathyroid adenoma. 
the adenoma and the severity of the symptoms, the presence of parathyroid crisis and mortality. However, this patient presented mild symptoms of hypercalcemia despite having a $10.6 \mathrm{~g}$ giant adenoma. In symptomatic patients, the most appropriate management is surgical resection of the affected gland. Guided by imaging studies such as sonography or Technetium-99 m scintigraphy, it is possible to accurately locate the adenoma and determine if there is involvement of more than one gland, or if the affected gland is located ectopically. Parathyroid surgery has evolved significantly in the past decades, and we now have minimally invasive techniques, which include a selective approach to the affected gland, together with intraoperative measurement of parathyroid hormone. Resection of the adenoma results in a total cure of the HPTP in 95\% of cases. Postoperative management consists of prophylaxis of "hungry bones syndrome" with calcium supplementation, as this is the most frequent complication after resection of a parathyroid adenoma due to transient hypocalcemia. Patients usually recover with no long-term complications or recurrence [4-7].

\section{Conclusion}

It is of great importance for physicians to have awareness that while most giant parathyroid adenomas tend to present higher levels of calcium and PTH, there seems to be a lower incidence of symptoms despite the hypercalcemic state, and therefore should be promptly considered in a mildly symptomatic patient with hypercalcemia. Additionally, these patients are more likely to develop symptomatic hypocalcemia after the resection of the adenoma. Furthermore, although only a small percentage of patients with nephrolithiasis have primary hyperparathyroidism, screening for hyperparathyroidism is still recommended. Imaging studies are of utmost importance when differentiating a giant adenoma and parathyroid cancerous tumor. It's important to note that the incidence of PHPT is higher in postmenopausal women compared to men of a similar age, while both genders seem to be equally affected at younger ages. This case also supports the effectiveness of minimally invasive techniques, which include a selective approach to the affected gland, together with intraoperative measurement of parathyroid hormone, as it is effective, less invasive, and precise.

\section{Acknowledgements}

All authors involved approved the final manuscript as submitted and agree to be accountable for all aspects of the work. We received no financial compensation for this case report.

\section{References}

1. Noureldine SI, Gooi Z, Tufano RP (2015) Minimally invasive parathyroid surgery. Gland surgery 4: 410-419.

2. Parathyroidectomy Minimally Invasive (focused). UCLA Endocrine Center. UCLA Health.

3. Casas Rodera P, Delgado-Ureña JG, Bernáldez Millán R (2021) Cirugía De Las Glándulas Paratiroides. Libro virtual de formación en ORL. Hospital universitario La Paz, Madrid.

4. Al-Hassan MS, Mekhaimar M, Ansari WE, et al. (2019) Giant parathyroid adenoma: A case report and review of the literature. Journal of Medical Case Reports 13: 332.

5. (2021) Primary Hyperparathyroidism. National Institute of Diabetes and Digestive and Kidney Diseases. U.S. Department of Health and Human Services.

6. Grover M, Sharma M, Bhandari S, et al. (2020) Giant parathyroid adenoma: Are they different? Indian Journal of Otolaryngology and Head \& Neck Surgery.

7. Cuenca D, Peña JF, Mercado M (2012) Adenoma gigante de paratiroides: Presentación de un caso. Revista de Endocrinología y Nutrición 20: 88-91.

DOI: $10.36959 / 608 / 456$ 